

\title{
Control parameter for the glass transition of glycerol evidenced by the static-field-induced nonlinear response
}

D L 'Hôte, R Tourbot, François Ladieu, P Gadige

\section{To cite this version:}

D L 'Hôte, R Tourbot, François Ladieu, P Gadige. Control parameter for the glass transition of glycerol evidenced by the static-field-induced nonlinear response. Physical Review B: Condensed Matter and Materials Physics (1998-2015), 2014, 90, pp.104202. 10.1103/PhysRevB.90.104202 . cea-01384225

\section{HAL Id: cea-01384225 \\ https://hal-cea.archives-ouvertes.fr/cea-01384225}

Submitted on 19 Oct 2016

HAL is a multi-disciplinary open access archive for the deposit and dissemination of scientific research documents, whether they are published or not. The documents may come from teaching and research institutions in France or abroad, or from public or private research centers.
L'archive ouverte pluridisciplinaire HAL, est destinée au dépôt et à la diffusion de documents scientifiques de niveau recherche, publiés ou non, émanant des établissements d'enseignement et de recherche français ou étrangers, des laboratoires publics ou privés. 


\title{
Control parameter for the glass transition of glycerol evidenced by the static-field-induced nonlinear response
}

\author{
D. L'Hôte, ${ }^{*}$ R. Tourbot, F. Ladieu, ${ }^{\dagger}$ and P. Gadige \\ SPEC/SPHYNX (CNRS URA 2464), DSM/IRAMIS CEA Saclay, Bat. 772, F-91191 Gif-sur-Yvette, France
}

(Received 21 December 2013; revised manuscript received 9 July 2014; published 8 September 2014)

\begin{abstract}
By studying a nonlinear susceptibility on supercooled glycerol, we show that applying a static field $E_{s t}$ increases the glass transition temperature $T_{g}$ by an amount quadratic in $E_{s t}$. This has important consequences: (i) it reinforces the relation between the two paths put forward in the last years to unveil the dynamical correlation volume close to $T_{g}$; (ii) it clarifies the interpretation of nonlinear measurements; (iii) it yields a new control parameter of the glass transition, which paves the way for experiments deepening our understanding of glasses.
\end{abstract}

DOI: 10.1103/PhysRevB.90.104202

PACS number(s): 64.70.kj, 77.22.-d, 89.75.Da

\section{INTRODUCTION}

Many materials of industrial importance are glasses, from window glasses and plastic bottles to emulsions, foams, dense assemblies of grains, and so on. Understanding the formation of these amorphous solids, the so-called glass and jamming transitions, is still a challenge, despite a large research effort over the last decades [1]. All these systems exhibit a remarkable "viscous slowing down" phenomenon, i.e., a dramatic increase of the relaxation time $\tau_{\alpha}$ when varying the control parameter by a modest amount. Although there is some evidence that jamming and glass transitions correspond to different physical phenomena [2], it was shown [3-5] in both cases that, close enough to the transition, relaxation occurs by groups of $N_{\text {corr }}$ dynamically correlated particles, with some groups being faster than others [hence the name dynamical heterogeneities (DH) for these groups]. However, the underlying mechanism of such a heterogeneous dynamics remains controversial [3-5]. For supercooled liquids, according to some approaches [6,7] the drainage of free volume when density increases is the main reason for the strong increase of $\tau_{\alpha}$ when lowering the temperature $T$ towards the glass transition temperature $T_{g}$. At the other extreme, another scenario relies on thermally activated processes on a constant-density energy landscape [8-10]. This has motivated significant experimental efforts [11-15] where $\tau_{\alpha}$ has been monitored both as a function of temperature $T$ and pressure $\Pi$, to assess which is the dominant control parameter of the glass transition.

Here we report the existence of a new control parameter of the glass transition. Namely, we show that applying a static electrical field $E_{s t}$ onto a prototypical glass forming liquid-glycerol—is equivalent to increasing $T_{g}$ by an amount $\delta T_{g}\left(E_{s t}\right) \propto E_{s t}^{2}$. Even though such an effect is anticipated in the framework of mode coupling theory (MCT) [16], this is the first experimental evidence of the influence of a static electric field on $T_{g}$. This is not surprising owing to the smallness of the effect that we report here $\left(\delta T_{g} \simeq 0.3 \mathrm{mK}\right.$ for $E_{s t}=1 \mathrm{MV} / \mathrm{m}$ ). Even if this is much smaller than the shifts of $T_{g}$ reported when varying $\Pi$, we shall show that the $T_{g}\left(E_{s t}\right)$ results reported here have important physical consequences-in the same way as the $T_{g}(\Pi)$ dependence

\footnotetext{
*deceased

†francois.ladieu@cea.fr
}

gives a deeper insight about the glass transition [11-15]. First, our results establish a quantitative link between the two independent methods put forward theoretically to relate $N_{\text {corr }}$ to macroscopic observables [17-19]. This link is of great importance since it deepens the overall consistency of the recent progress made about the characterization of the DH's. Second, our results put a severe constraint on the phenomenological models of the nonlinear response, which clarifies the interpretation of nonlinear measurements. Third, the fact that $E_{s t}$ is a control parameter opens the way for new experiments, deepening our understanding of glasses.

The paper is organized as follows. We first define and explain in Sec. II how we measure the nonlinear susceptibility $\chi_{2 ; 1}^{(1)}$ associated to a static field. Then, in Sec. III, we show the behavior of $\chi_{2: 1}^{(1)}$ and why it unveils the above-mentioned shift of $T_{g}$ with $E_{s t}$. Section IV is devoted to the discussion of the main physical consequences of our experiments. Finally, in Sec. V, we give some perspectives and summarize our work.

\section{EXPERIMENTS}

\section{A. Definitions}

In a closed cell, ultrapure glycerol was put between stainless steel electrodes and submitted to an electric field $E$. The resulting polarization $P$ was overwhelmingly dominated by its linear component $P_{\text {Lin }}$. We recall that, for an ac field $E_{a c} \cos (\omega t)$ oscillating as a function of the time $t$ with the frequency $f=\omega /(2 \pi)$, the complex linear susceptibility $\chi_{\text {Lin }}=\chi_{\text {Lin }}^{\prime}-i \chi_{\text {Lin }}^{\prime \prime}\left(\right.$ with $\left.i^{2}=-1\right)$ is defined by $P_{\text {Lin }}=$ $\epsilon_{0} E_{a c}\left[\chi_{\operatorname{Lin}}^{\prime} \cos (\omega t)+\chi_{\operatorname{Lin}}^{\prime \prime} \sin (\omega t)\right]$ where $\epsilon_{0}$ is the vacuum dielectric constant. At a given $T, \chi_{\text {Lin }}^{\prime \prime}$ is peaked at a frequency $f_{\alpha}$, which yields the relaxation time $\tau_{\alpha} \equiv 1 /\left(2 \pi f_{\alpha}\right)$. As detailed in Refs. [20-22] (see also Fig. 1), in our "two samples bridge" $P_{\text {Lin }}$ cancels very efficiently. This allows to study the nonlinear polarization $\delta P \equiv P-P_{\text {Lin }}$ at any $f$, at variance with other setups [23-25]. For an isotropic liquid submitted to an external electric field $E(t)$, the most general expression of the nonlinear polarization $\delta P(t)$ is given by a series expansion in $E(t)$ [note that no even terms are allowed because of the symmetry with respect to field reversal $E(t) \rightarrow-E(t)$ ]:

$$
\begin{aligned}
\frac{\delta P(t)}{\epsilon_{0}}= & \iiint_{-\infty}^{\infty} \chi_{3}\left(t-t_{1}^{\prime}, t-t_{2}^{\prime}, t-t_{3}^{\prime}\right) \\
& \times E\left(t_{1}^{\prime}\right) E\left(t_{2}^{\prime}\right) E\left(t_{3}^{\prime}\right) d t_{1}^{\prime} d t_{2}^{\prime} d t_{3}^{\prime}+\cdots .
\end{aligned}
$$




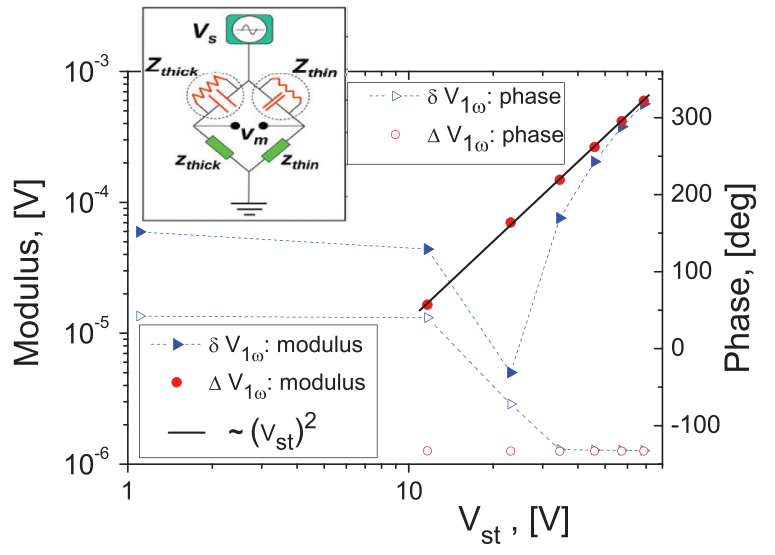

FIG. 1. (Color online) Inset: Representation of our "two samples bridge": the source voltage $V_{s}(t)$ is the sum of a constant voltage $V_{s t}$ and of an ac voltage $V_{a c} \cos (\omega t)$. The left (right) branch contains the thick (thin) sample of impedance $Z_{\text {thick }}\left(Z_{\text {thin }}\right)$. With a lock-in amplifier, we measure the $1 \omega$ component $\delta V_{1 \omega}$ of the differential voltage $V_{m}$ between the two middle points of each arm of the bridge, after having tuned the values of the impedances $z_{\text {thin }}$ and $z_{\text {thick }}$ so as to suppress accurately the linear voltage coming from the linear response $P_{\text {Lin }}$. Main panel: Example of the rms values of $\delta V_{1 \omega}\left(V_{a c}, V_{s t}\right)$ measured in the stationary regime (see text) by varying $V_{s t}$ and keeping constant $V_{a c}$. To get accurate values of the quantity of interest $\Delta V_{1 \omega}\left(V_{a c}, V_{s t}\right)=\delta V_{1 \omega}\left(V_{a c}, V_{s t}\right)-\delta V_{1 \omega}\left(V_{a c}, 0\right)$, we measure the stationary regime value of $\delta V_{1 \omega}\left(V_{a c}, 0\right)$ before switching on and after switching off $V_{s t}$. We average these two values of $\delta V_{1 \omega}\left(V_{a c}, 0\right)$ and subtract this average to $\delta V_{1 \omega}\left(V_{a c}, V_{s t}\right)$ to get the sought $\Delta V_{1 \omega}\left(V_{a c}, V_{s t}\right)$ which is directly proportional to $\chi_{2 ; 1}^{(1)}$. One observes on the graph that $\Delta V_{1 \omega}\left(V_{a c}, V_{s t}\right)$ has a modulus proportional to $V_{s t}^{2}$ (the thick solid line is such a quadratic dependence) and a phase independent on $V_{s t}$, in good agreement with Eq. (2). Note that these two key features are not observed for the values of $\delta V_{1 \omega}\left(V_{a c}, V_{s t}\right)$-a single value of $\delta V_{1 \omega}\left(V_{a c}, 0\right)$ is plotted on the graph at an arbitrary value of $V_{s t}=1.1 \mathrm{~V}-$. The data plotted here were obtained in the following conditions: the thickness of the thin (thick) sample is $16.8 \mu \mathrm{m}(46.2 \mu \mathrm{m}) ; T=197 \mathrm{~K} ; f=0.8 \mathrm{~Hz} \simeq 3 f_{\alpha}$, where $f_{\alpha}$ is the relaxation frequency; $z_{\text {thin }} \simeq 3.56 \mathrm{M} \Omega ; z_{\text {thick }} \simeq 9.9 \mathrm{M} \Omega$; $V_{a c}=7.84 \mathrm{~V}_{\mathrm{rms}}, S=2.27 \mathrm{~cm}^{2}$ where $S$ is the surface of each sample. To make the connection between measured voltage and polarization, one has to use the standard complex impedance relating voltage to the intensity $I$, as well as the relation $I=S \partial P / \partial t$, see, e.g., Ref. [20].

For the fields used here, $\delta P$ is overwhelmingly dominated by the cubic response $\left(\propto E^{3}\right)$, this is why the higher-order terms proportionnal to $E^{5}, E^{7}, \ldots$, are not written explicitly in Eq. (1). All the nonlinear cubic responses come from Eq. (1) and can be characterized by the triple Fourier transform kernel $\chi_{3}\left(\omega_{1}, \omega_{2}, \omega_{3}\right)$. For a pure ac field $E_{a c}, \delta P$ contains two terms studied in Refs. [20-22]. The first one oscillates at $1 \omega$ and is given by $\delta P_{1 \omega}\left(E_{a c}\right)=\frac{3}{4} \epsilon_{0} E_{a c}^{3}\left|\chi_{3}^{(1)}\right| \cos \left(\omega t-\delta_{3}^{(1)}\right)$ where we have noticed the corresponding cubic susceptibility [22] by $\chi_{3}^{(1)}$ instead of the more heavy notation $\chi_{3}(-\omega, \omega, \omega)$. The second one $\propto \chi_{3}(\omega, \omega, \omega) E_{a c}^{3}$ oscillates at $3 \omega$, and similarly $[20,21]$, we call the corresponding cubic susceptibility $\chi_{3}^{(3)}$. In the present work, a static field $E_{s t}$ was applied on top of an ac field $E_{a c}$. This yields a series of new cubic terms in $\delta P$, e.g., terms proportional to $E_{a c}^{2} E_{s t}$ or to $E_{a c} E_{s t}^{2}$. Here we focus on the new cubic term $\Delta P_{1 \omega}$, which appears due to the static field in the $1 \omega$ component of $\delta P$. Since $\Delta P_{1 \omega} \equiv \delta P_{1 \omega}\left(E_{a c}, E_{s t}\right)-$ $\delta P_{1 \omega}\left(E_{a c}, 0\right)$, where we recall that $\delta P_{1 \omega}\left(E_{a c}, 0\right)$ is the term proportional to $\chi_{3}^{(1)}$ mentionned above, one gets

$$
\Delta P_{1 \omega}=3 \epsilon_{0} E_{a c} E_{s t}^{2}\left|\chi_{2 ; 1}^{(1)}\right| \cos \left(\omega t-\delta_{2 ; 1}^{(1)}\right),
$$

where $\chi_{2 ; 1}^{(1)}$ stands for $\chi_{3}(0,0, \omega)$, and $-\delta_{2 ; 1}^{(1)}$ is its phase.

\section{B. Measurement of $\chi_{2 ; 1}^{(1)}$}

In our experiment $E_{a c}$ is kept constant, and thanks to our bridge setup described in Fig. 1, we monitor directly the first harmonics component $\delta P_{1 \omega}$ of $\delta P$ for various values of $E_{s t}$. We then form the difference $\Delta P_{1 \omega}=\delta P_{1 \omega}\left(E_{a c}, E_{s t}\right)-$ $\delta P_{1 \omega}\left(E_{a c}, 0\right)$. As a result $\Delta P_{1 \omega}$ is directly proportional to the sought $\chi_{2 ; 1}^{(1)}$. More precisely, for each given values of $T, f$, we first measure the value of $\delta P_{1 \omega}\left(E_{a c}, 0\right)$, then the static field is switched on to a given $E_{s t}$ and we wait for a time $\tau_{s t}$, long enough to let all the transients vanish, before measuring $\delta P_{1 \omega}\left(E_{a c}, E_{s t}\right)$. Then we switch off $E_{s t}$ and measure again the stationary regime value of $\delta P_{1 \omega}\left(E_{a c}, 0\right)$, average it with the value measured before switching on $E_{s t}$, subtract this average to $\delta P_{1 \omega}\left(E_{a c}, E_{s t}\right)$, and obtain the sought $\Delta P_{1 \omega}$. We then do again this sequence with the opposite sign of $E_{s t}: \Delta P_{1 \omega}$ is measured again, with the same delay of $\tau_{s t}$. This inversion allows both to check that our measurements do not depend on the sign of $E_{s t}$, as expected from Eq. (2), and to avoid any spurious electrode charging effects coming from any slow drift of the small amount of free ions in the samples. For a significant number of points, by increasing the value of $\tau_{s t}$ by a factor of 4 , we checked that $\tau_{s t}$ was long enough so as to have no influence on our measurements. This is very important since it shows that our results are really in the stationary regime, i.e., they are not affected by the transient effects coming from the switching on of the static field. In practice, $f \tau_{s t}$ and $f_{\alpha} \tau_{s t}$ were typically much larger than 10, the minimal values. Finally, we checked that $\Delta P_{1 \omega}$ was proportional to $E_{a c}$. Our "two samples bridge" setup has already been depicted in Refs. [20-22], but for the sake of clarity, and owing to the fact that $\chi_{2 ; 1}^{(1)}$ has never been reported before, all the key information is given in the caption of Fig. 1, hence its unusual length. Typically, in the thinnest sample $E_{a c}=0.65 \mathrm{MV} / \mathrm{m}$, and $0 \leqslant E_{s t} \leqslant 4.1 \mathrm{MV} / \mathrm{m}$. We emphasize that, in Fig. 1, the analysis of $\Delta P_{1 \omega}$ as a function of $E_{s t}$ yields the quadratic dependence expected from Eq. (2), giving $\chi_{2 ; 1}^{(1)}$ with a $\pm 4 \%$ error bar.

\section{RESULTS}

In the previous works [26-28] devoted to the effects of pressure $\Pi$, the shift $\delta T_{g}(\Pi)$ of $T_{g}$ is obtained by superimposing the polarization obtained at two different pressures. We follow this rule here: Stating that $E_{s t}$ shifts $T_{g}$ by $\delta T_{g}\left(E_{s t}\right)$ is equivalent to fulfill the equation $P_{1 \omega}\left(E_{a c}, E_{s t}, T\right)=$ $P_{1 \omega}\left[E_{a c}, 0, T-\delta T_{g}\left(E_{s t}\right)\right]$. Note that, as pressure experiments are done with a pure ac field $E_{a c}, \delta T_{g}(\Pi)$ is the solution of $P\left(E_{a c}, \Pi, T\right)=P\left[E_{a c}, 0, T-\delta T_{g}(\Pi)\right]$. Here the corresponding equation must be written on the odd harmonics only since $E_{s t}$ generates even harmonics $(0 \omega, 2 \omega)$ which do not exist when $E_{s t}=0$. This is why we solve the equation on $P_{1 \omega}$, 


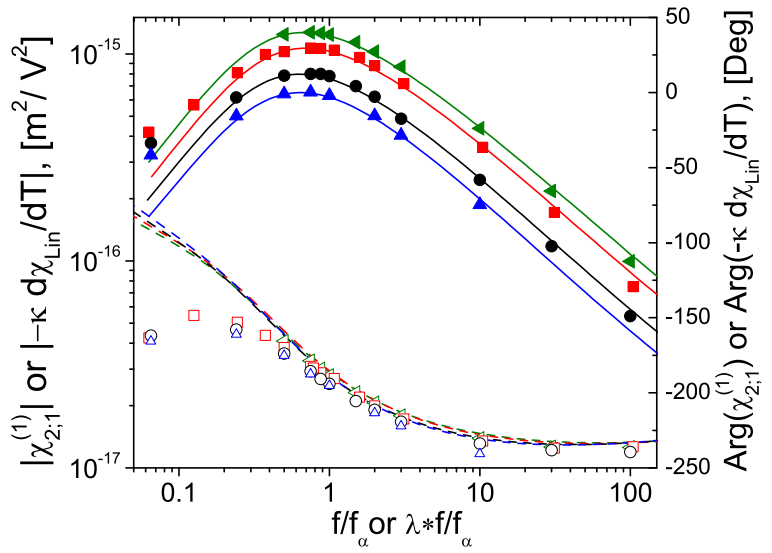

FIG. 2. (Color online) Comparison of $\chi_{2 ; 1}^{(1)}$ (symbols) and of $-\kappa\left(\partial \chi_{\text {Lin }} / \partial T\right)$ (lines, shifted horizontally by a factor $\left.\lambda_{\text {eff }} \simeq 0.80\right)$. Left axis: Moduli (full symbols or plain lines). From top to bottom $T=197,202,211$, and $218 \mathrm{~K}$, which amounts, at $E_{s t}=0$, to $f_{\alpha}=$ $0.27,2.1,60$, and $520 \mathrm{~Hz}$. Right axis: Phases (open symbols or dashed lines). Here $\lambda_{\text {eff }}=0.80 \pm 0.04, \kappa=(1.18 \pm 0.03) \times 10^{-16} \mathrm{Km}^{2} / \mathrm{V}^{2}$. Symbols are larger than the error bar: $\pm 4 \%$ for $\left|\chi_{2 ; 1}^{(1)}\right|$ and \pm 4 degrees for its phase.

the similar one on the $3 \omega$ component of $P$ requires to measure five order responses, i.e., a more sensitive setup. For symmetry reasons we set $\delta T_{g}=3 \kappa E_{s t}^{2}$ with $\kappa$ a constant. As we shall find $\delta T_{g}\left(E_{s t}\right) \ll T$, and as $P$ is overwhelmingly dominated by $P_{\text {Lin }}$, Eq. (3) below is obtained by the following: (i) lowest-order expansion of $P_{\mathrm{Lin}}\left(E_{a c}, 0, T-3 \kappa E_{s t}^{2}\right)$; (ii) identification of the expansion with Eq. (2). This is how we obtain

$$
\chi_{2 ; 1}^{(1)}(f, T)=-\kappa\left(\frac{\partial \chi_{\mathrm{Lin}}}{\partial T}\right)_{f / \lambda_{\mathrm{eff}}, T, E_{s t}=0},
$$

where the introduction of the effective parameter $\lambda_{\text {eff }}$ equal to 1 in the simplest case-is justified below.

Figure 2 shows that, for our data, Eq. (3) holds, as soon as $f / f_{\alpha} \geqslant 0.2$, with $\lambda_{\text {eff }} \simeq 0.80$ and $\kappa \simeq 1.18 \times 10^{-16} \mathrm{Km}^{2} / \mathrm{V}^{2}$. Figure 2 corresponds to a $T$ range where $\tau_{\alpha}$ varies by more than three decades: in this range, $\kappa$ and $\lambda_{\text {eff }}$ may slightly increase

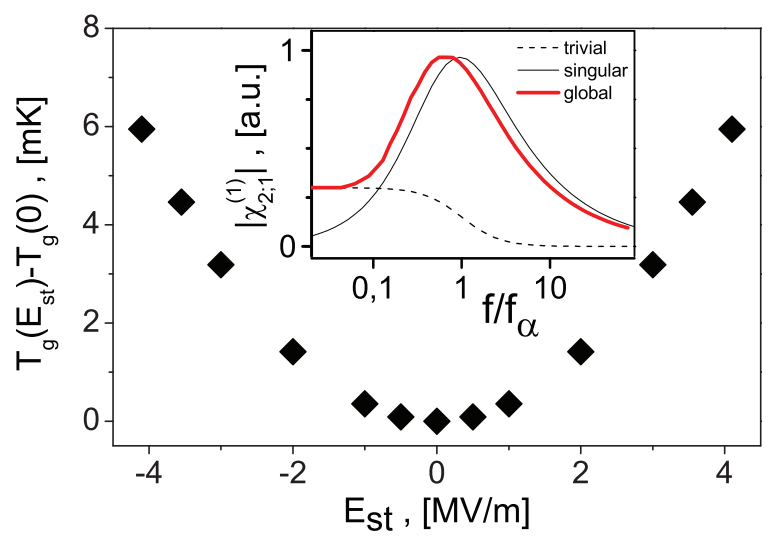

FIG. 3. (Color online) Inset: Qualitative picture of the trivial, singular, and global $\chi_{2 ; 1}^{(1)}$. Main panel: Evolution of $T_{g}$ with $E_{s t}$ by the quantity $3 \kappa E_{s t}^{2}$. The size of the points is larger than the $\pm 2.5 \%$ uncertainty on $\kappa$.

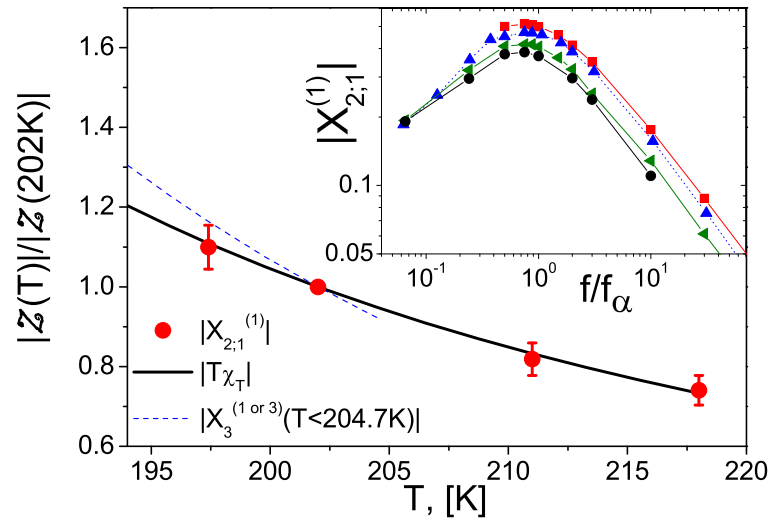

FIG. 4. (Color online) Inset: Measured dimensionless susceptibility $X_{2 ; 1}^{(1)}$ (same $T^{\prime}$ s as in Fig. 2). Main panel: $T$ dependencies of various quantities $\mathcal{Z}$ normalized to 1 at $202 \mathrm{~K}$.

when lowering $T$ but this possible effect is extremely tiny since it is close to the experimental uncertainty (namely $\pm 2.5 \%$ on $\kappa$ and $\pm 5 \%$ on $\lambda_{\text {eff }}$ ). We emphasize that our data obey Eq. (3) both for the moduli and for the phases, which is highly nontrivial. By contrast, keeping $\lambda_{\text {eff }}$ reasonably close to 1 , one finds $[21,22]$ that Eq. (3) holds neither for $\chi_{3}^{(1)}$ nor for $\chi_{3}^{(3)}$.

Let us now briefly explain why for our data $\lambda_{\text {eff }}$ is not strictly equal to 1 . We first recall, see Fig. 2, that Eq. (3) fails for our data at low values of $f / f_{\alpha}$, i.e., for time scales larger than $\tau_{\alpha}$, over which the liquid flows: this destroys the glassy correlations, and makes any molecule effectively independent of others [1,4]. When $f \ll f_{\alpha}$, we thus expect a "trivial" physics, resembling that of an ideal gas: the corresponding $\left|\chi_{2 ; 1}^{(1)}\right|_{\text {triv }}$ is depicted as a dashed line [29] in the inset of Fig. 3. It is qualitatively different from the "singular" $\left|\chi_{2 ; 1}^{(1)}\right|_{\text {sing }}$ [thin solid line in the inset of Fig. 3] that one obtains when the glassy correlations dominate. Increasing $f$ from $f \ll f_{\alpha}$, we thus expect a crossover between $\left|\chi_{2 ; 1}^{(1)}\right|_{\text {triv }}$ and $\left|\chi_{2 ; 1}^{(1)}\right|_{\text {sing }}$, yielding the "global" $\left|\chi_{2 ; 1}^{(1)}\right|$ : the crossover distorts $\left|\chi_{2 ; 1}^{(1)}\right|$ even close to $f_{\alpha}$, which may slightly shift its peak-see the thick solid line in the inset of Fig. 3. As this crossover has little impact on $\partial \chi_{\operatorname{lin}} / \partial T$, which is in any case peaked around $f_{\alpha}$, we thus expect that a factor $\lambda_{\text {eff }}$ close to 1 is needed in Eq. (3) to compensate for the small shift of $\chi_{2 ; 1}^{(1)}$. This reasoning is reinforced by several facts: first, in MCT [16] one recovers Eq. (3) with $\lambda_{\text {eff }}=1$ consistently with the divergence of the singular response which rejects the crossover to $f / f_{\alpha} \rightarrow 0$, see also Ref. [30]. Second in the inset of Fig. 4, one sees that, in the limit $f / f_{\alpha} \ll 1, X_{2 ; 1}^{(1)}$ (defined below) becomes $T$ independent, which is characteristic of an ideal gas [31]. Third, similar considerations have already been elaborated in Refs. [21,22] for the low frequency behavior of $\chi_{3}^{(1 \text { or 3). }}$.

\section{DISCUSSION}

\section{A. Physical picture}

Having qualitatively accounted for the limits of Eq. (3) close to $T_{g}$, we interpret our data as reflecting the fact that $E_{s t}$ is a new control parameter of the glass transition since it shifts $T_{g}$ by an amount $3 \kappa E_{s t}^{2}$, as illustrated in Fig. 3. To give a 
physical picture, we recall that translation and rotation are well coupled in glycerol [32], and that dynamical correlations do not correspond to any simple spatial order $[1,3]$. As $E_{s t}$ tends to rotate the molecules and align their polarization vectors, it also alters their positions because molecules have a given shape and rotating them conflicts with steric constraints: $E_{s t}$ thus corresponds to a new constraint unfavorable to dynamical correlations, i.e., it slows down the dynamics, hence the increase of $T_{g}$ with $E_{s t}$. More generally, a static field gives rise to subtle effects strongly dependent of the kind of frustrated system which is considered: in the very specific case of spin glasses, the critical temperature may decrease with the static (magnetic) field (although this is strongly debated [33-36]), while in other systems reentrant behaviors at very high fields may happen [37]. Some further work is in progress to study the behavior of $\delta T_{g}\left(E_{s t}\right)$ at higher values of the static field.

\section{B. Relating nonlinear cubic responses and $T \chi_{T}$}

\section{Frequency dependence}

The fact that our data of Fig. 2 follow Eq. (3) is the first experimental evidence that (some) nonlinear responses can be tightly related to $T \chi_{T} \equiv T \partial\left(\chi_{\text {Lin }} / \Delta \chi_{1}\right) / \partial T$ where $\Delta \chi_{1}=\chi_{\text {Lin }}(0)-\chi_{\text {Lin }}(\infty)$. This is qualitatively important since two lines of thought have related the same number $N_{\text {corr }}$ of dynamically correlated molecules either to the cubic susceptibility $\chi_{3}^{(3)}$ (see Ref. [17]) or to $\operatorname{Max}_{f}\left|T \chi_{T}\right|$ (see Refs. $[18,19])$. Up to now, the only experimental indication of the compatibility of these two approaches is that the $T$ dependence of the dimensionless version $X_{3}^{(3)}$ of $\chi_{3}^{(3)}$ is close to that of $\operatorname{Max}_{f}\left|T \chi_{T}\right|$, see Fig. 4 and Refs. [21,22,38,39]. The fact that the frequency dependence of a cubic susceptibility $\left[\chi_{2 ; 1}^{(1)}\right]$ can be mapped to that of $T \chi_{T}$ on a wide frequency interval is a new result which strongly reinforces the compatibility of the two theoretical approaches.

\section{Temperature dependence}

To get the $T$ dependence of $N_{\text {corr }}$ precisely [17], the cubic susceptibilities $\chi_{n}^{(k)}$, with $n=3$ or " 2 ; 1 " and $k=$ 1 or 3 , must be put in their dimensionless form $X_{n}^{(k)}=$ $\chi_{n}^{(k)}\left\{k_{B} T /\left[\epsilon_{0}\left(\Delta \chi_{1}\right)^{2} a^{3}\right]\right\}$ where $a^{3}$ is the molecular volume and $k_{B}$ the Boltzmann constant. As evoked above, in the trivial case of an ideal gas the various $\left|X_{n}^{(k)}\left(f / f_{\alpha}\right)\right|$ are $T$ independent [29], while in a supercooled liquid, for $n=3$, they are proportional to $N_{\text {corr }}(T)$ according to Refs. [17,21,22,38]. In Fig. 4 all the observables $\mathcal{Z}(T)$ are normalized at $202 \mathrm{~K}$ : (i) the thick line corresponds to the $\operatorname{Max}_{f}\left|T \chi_{T}\right|$ which was found in Refs. [21,38] to be very close to the $T$ dependence of the maximum over the frequency of $\left|X_{3}^{(3)}\right|$; (ii) the thin dashed line corresponds to the best estimate of $N_{\text {corr }}(T<204.7 \mathrm{~K})$, drawn from the refined analysis of Ref. [22] comparing $\left|X_{3}^{(3)}\right|$ and $\left|X_{3}^{(1)}\right|$; (iii) the points show the $T$ dependence of $\left|X_{2 ; 1}^{(1)}\right|$ drawn [40] from the inset of Fig. 4. Figure 4 shows that the various $\left|X_{n}^{(k)}\right|$ have mainly the same $T$ dependence. This result goes beyond the prediction of Ref. [17], where the effect of $E_{s t}$ was not studied, and had been anticipated only within MCT [16].

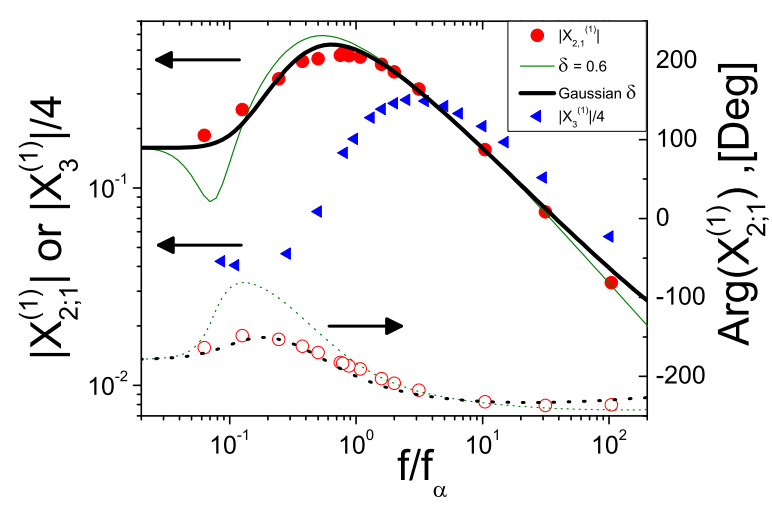

FIG. 5. (Color online) At $202 \mathrm{~K}$, modulus (left axis) and phase (right axis) of measured dimensionless susceptibilities (symbols). Lines are fits of $X_{2 ; 1}^{(1)}$ with the toy model of Ref. [47] by choosing either $N_{\text {corr }}=16$ and $\delta=0.60$; or $N_{\text {corr }}=10$ and $\delta$ distributed onto $[0 ; 1]$ as $\propto \exp \left[-\left(\delta-\delta_{1}\right)^{2} /\left(2 \delta_{2}^{2}\right)\right]$ with $\delta_{1}=0.30$ and $\delta_{1}=0.29$, see the Appendix.

\section{Constraints put onto phenomenological models}

\section{Box model}

Even though our results deepens the consistency of using cubic susceptibilities to get the $T$ dependence of $N_{\text {corr }}$, a problem is still pending. Indeed, a phenomenological model, called the "box model" [42-44] has been able to account fairly well (see Ref. [41]) for the nonlinear experiments published up to now despite it assumes that the nonlinear behavior comes from the power $\mathscr{P}$ dissipated by the electric field in each DH: this yields, finally, $\chi_{3}^{(k)}$ to be independent of $N_{\text {corr }}$. Our results show that the dissipated power $\mathscr{P}$ is not the main source of $\chi_{2 ; 1}^{(1)}$. Indeed, as our experiments are in the stationary regime, varying $E_{s t}$ does not change the power dissipated by the dipoles, and $\mathscr{P}$ only varies due to the small dc conductivity $\sigma$ coming from the small amount of mobile ions in the liquid. It turns out, see the Appendix, that $\sigma$ is smaller by a factor 300 than $\epsilon_{0} \omega_{\alpha} \chi_{\text {Lin }}^{\prime \prime}\left(f_{\alpha}\right)$, which is the corresponding quantity when measuring $\chi_{3}^{(1)}\left(f_{\alpha}\right)$. As the box model fairly well accounts for $\chi_{3}^{(1)}\left(f>f_{\alpha}\right)$, we predict within this model, for $f \geqslant f_{\alpha}$, values of $\left|X_{2 ; 1}^{(1)}\right|$ which are orders of magnitude smaller than $\left|X_{3}^{(1)}\right|$-see the Appendix for a detailed calculation. This is contradicted by Fig. 5, where $\left|X_{2 ; 1}^{(1)}\right|$ has the same order of magnitude as $\left|X_{3}^{(1)} / 4\right|$ - the factor of 4 is due to the precise definitions of $\chi_{3}^{(1)}$ and of $\chi_{2 ; 1}^{(1)}$ given in Sec. II A. Thus, for the first time, the box model is unable to account for a nonlinear experiment, namely $\chi_{2 ; 1}^{(1)}$. This is an important step forward clarifying the interpretation of nonlinear responses.

\section{2. "Toy" model}

For completeness we now briefly evoke another phenomenological model, namely the "toy" model of Refs. [45-47], where each dynamical heterogeneity (DH) is modeled as a point evolving in a two well potential of (dimensionless) asymmetry $0 \leqslant \delta \leqslant 1$. A key assumption, added in Ref. [47], is that due to the absence of structural order, the net dipole moment of a given DH is proportional to $\sqrt{N_{\text {corr }}}$. This yields $X_{3}^{(k)} \propto N_{\text {corr }}$ and, with some reasonable values 
of the free parameters (mostly $N_{\text {corr }}$ and $\delta$ ), an acceptable agreement [47] for the frequency dependence of $X_{3}^{(k)}$ at $204.5 \mathrm{~K}$. For $f \geqslant f_{\alpha}$, we show in the Appendix that this model predicts the same $T$ dependence for $\left|X_{2 ; 1}^{(1)}\right|$ as for $\left|X_{3}^{(k)}\right|$ since all these cubic susceptibilities are found to be proportional to $N_{\text {corr }}(T)$, consistently with Fig. 4 . Besides, we show in Fig. 5 that the "toy" model can account for the frequency behavior of $X_{2 ; 1}^{(1)}$ : at $202 \mathrm{~K}$ we obtain a good fit with $\delta=0.60$ and $N_{\text {corr }}=16$, i.e., for parameters reasonably close to those used in Ref. [47] at $204.5 \mathrm{~K}$. The unphysical oscillation close to $0.1 f_{\alpha}$ comes from the heuristic combination of the DH's and of the trivial contribution: this disappears when assuming that $\delta$ is distributed among the various $\mathrm{DH}$, but this is at the cost of a supplementary parameter, see Fig. 5 . Note that the toy model does not recover exactly Eq. (3) with $\lambda_{\text {eff }}=1$ since, as shown in the Appendix, $\chi_{2 ; 1}^{(1)}$ contains two other-quite small-terms in addition to a term $\propto \partial \chi_{\text {Lin }} / \partial T$. Recovering Eq. (3) thus requires a deeper theoretical framework.

\section{PERSPECTIVES AND CONCLUSION}

Finally, our study opens the way for new experiments: due to the very small value of $\mathscr{P}$, one could draw the $N_{\text {corr }}(T)$ at high $T$ 's from $\chi_{2 ; 1}^{(1)}$, avoiding the heating problems which limit the reliability of $\chi_{3}^{(k)}$ when $f_{\alpha}$ is large enough [48]. Below $T_{g}$, by switching on $E_{s t}$ to $100 \mathrm{MV} / \mathrm{m}$ - the recipe to get this kind of high fields was found in Ref. [49]- $\delta T_{g}$ could be as large as $3 \mathrm{~K}$, which will increase $\tau_{\alpha}$ by typically a decade. One could thus trigger aging phenomena which could be studied from the early stages since switching on $E_{s t}$ is much faster than varying $T$. Having access to aging on a much larger number of decades in time will probably deepen our understanding of this phenomenon which is the hallmark of glasses.

To conclude, by monitoring a new kind of nonlinear susceptibility, we have shown that a static electric field $E_{s t}$ shifts $T_{g}$ in glycerol. We found evidence of a quantitative relation between the two theoretical paths relating $N_{\text {corr }}$ to macroscopic observables, clarified the interpretation of nonlinear susceptibilities close to $T_{g}$, and proposed experiments which should give hints about the physics of glasses.

\section{ACKNOWLEDGMENTS}

We thank A. Dehaoui for experimental help during his stay, and C. Alba-Simionesco, G. Biroli, J.-P. Bouchaud, P.M. Déjardin, and G. Tarjus for long-lasting help. The support of the european grant "NPRGGLASS" as well as the support of the Institut des Systemes Complexes de la Region Ile de France are warmly acknowledged.

\section{APPENDIX A: OVERESTIMATE OF THE BOX MODEL PREDICTION FOR $\chi_{2 ; 1}^{(1)}$}

In this section we shall compute nonlinear effects in the framework of the box model. As our experiments are in the stationary regime (see Sec. II B above), we do not compute the power dissipated by the switching on of the static field, but only consider the stationary dissipated power. We compare the case of a pure ac field $E_{a c}$, where one obtains $\chi_{3}^{(1)}$, with the case of a static field $E_{s t}$ (plus a fixed amplitude of $E_{a c}$ ) where one obtains $\chi_{2 ; 1}^{(1)}$ by varying $E_{s t} \cdot \chi_{3 ; \text { Box mod }}^{(1)}$ and $\chi_{2 ; 1 ; \text { Box mod }}^{(1)}$ will represent the predictions of the box model for $\chi_{3}^{(1)}$ and $\chi_{2 ; 1}^{(1)}$, respectively. As we shall find that $\chi_{2 ; 1 ; B o x}^{(1)}$ mod is by orders of magnitude smaller than $\chi_{3 \text {;Box mod }}^{(1)}$, it is sufficient to give an overestimate of dominant terms instead of performing a full calculation.

\section{Heatings produced by either $E_{a c}$ or $E_{s t}$}

In the box model, each dynamical heterogeneity (DH) is assumed to be heated by a quantity $\delta T$ due to the applied field, see Refs. [42,43].

(1) In the case of a pure ac field $E_{a c}$, one obtains [42]

$$
\delta T_{a c}=\frac{1}{2} \frac{\epsilon_{0} \Delta \chi_{1} E_{a c}^{2}}{c_{\mathrm{DH}}} \frac{\left(\omega \tau_{\mathrm{DH}}\right)^{2}}{1+\left(\omega \tau_{\mathrm{DH}}\right)^{2}} \simeq \frac{1}{2} \frac{\epsilon_{0} \Delta \chi_{1} E_{a c}^{2}}{c_{\mathrm{DH}}},
$$

where $c_{\mathrm{DH}}$ is the volumic specific heat of the DH's, while all other quantities are defined in the main text. In the last equality of Eq. (A1), we took into account that nonlinear effects are dominated by the DH's such that $\left(\omega \tau_{\mathrm{DH}}\right) \geqslant 1$. This happens for two reasons: (i) these kind of DH's are those for which $\delta T_{a c}$ reaches its maximum value; (ii) these DH's are the most numerous ones, as soon as $\omega \geqslant 1.12 \omega_{\alpha}$, with $\omega_{\alpha}=2 \pi f_{\alpha}$, see Ref. [44]. We neglect here the part of the heating oscillating at $2 \omega$ since it yields a subdominant contribution to the sought $\chi_{3 ; \text { Box mod }}^{(1)}$, see Ref. [43].

(2) In the case of an experiment measuring $\chi_{2 ; 1}^{(1)}$, the applied field is the sum of a static field and of an ac field. For the dipoles, on general grounds [48], the dissipated power is proportional to $(\partial P / \partial t)^{2}$. As, to the leading order, $P \propto \chi_{\text {Lin }}^{\prime}(\omega=$ $0) E_{s t}+\left|\chi_{\operatorname{Lin}}(\omega)\right| E_{a c} \cos \left\{\omega t-\arctan \left[\chi_{\text {Lin }}^{\prime \prime}(\omega) / \chi_{\text {Lin }}^{\prime}(\omega)\right]\right\}$, one finds that the power dissipated by the dipoles is only proportional to $E_{a c}^{2}$ and does not contain $E_{s t}$. Therefore, when varying $E_{s t}$ and keeping fixed $E_{a c}$, as we do in our experiments, the power dissipated by the dipoles does not change at all. This is at odds with what happens in an experiment measuring $\chi_{3}^{(1)}$ where the power dissipated by the dipoles is, according to the box model, the source of the cubic susceptibility. This is the main reason why we shall find below that within the box model one predicts $\left|\chi_{2 ; 1 ; \text { Box mod }}^{(1)}\right| \ll\left|\chi_{3 ; \text { Box mod }}^{(1)}\right|$.

In a $\chi_{2 ; 1}^{(1)}$ experiment, the dissipation in the liquid comes from the presence of a small quantity of free ions which yields a contribution $\chi_{\text {ions }}^{\prime \prime} \propto 1 / \omega$ : this contribution becomes visible only at very low frequencies, i.e., below an angular frequency $\omega_{\text {ions }}=t \omega_{\alpha}$ with $t \leqslant 2.5 \times 10^{-2}$, the actual value of $t$ depending of the purity of the supercooled liquid (glycerol here). It is convenient to write $\chi_{\text {ions }}^{\prime \prime}=s \Delta \chi_{1} \omega_{\text {ions }} / \omega$ where $s \leqslant 4 \times 10^{-2}$ is a very small number, the actual value of which again depends on the purity of the liquid. Below $\omega_{\text {ions }}$, the fact that $\chi^{\prime \prime} \propto 1 / \omega$ amounts to a conductivity $\sigma\left(\omega \leqslant \omega_{\text {ions }}\right)=$ $\epsilon_{0} \chi_{\text {ions }}^{\prime \prime} \omega$ which is independent on frequency. The dissipated power is thus

$$
\begin{aligned}
\mathscr{P} & =\sigma\left(E_{s t}+E_{a c}\right)^{2} \\
& =\sigma E_{s t}^{2}+2 \sigma E_{s t} E_{a c} \cos (\omega t)+\sigma E_{a c}^{2} \cos ^{2}(\omega t),
\end{aligned}
$$

The right-hand side of Eq. (A2) contains three terms. The term proportional to $E_{a c}^{2}$ has to be discarded since $E_{a c}$ is 
kept fixed in a $\chi_{2 ; 1}^{(1)}$ experiment (this is the same reason as above for the power dissipated by the dipoles). The term proportional to $E_{s t} E_{a c}$ oscillates at $1 \omega$ and gives a nonlinear polarization at $1 \omega$ by modulating the ions conductivity term $\propto \sigma E_{s t}$. However, this term is not the leading one since it involves a factor $(s t)^{2} \ll 1$, contrary to the term that we calculate now and which is proportional to $s t$ [see below Eq. (A8)]. Indeed, focusing on the first term of Eq. (A2), we get, with $\sigma=\epsilon_{0} \Delta \chi_{1} \omega_{\alpha} t s$, that the dominant contribution to the first harmonics nonlinear polarization comes from the term $\mathscr{P}_{s t}$ corresponding to the first term of the right-hand side of Eq. (A2):

$$
\mathscr{P}_{s t}=\sigma E_{s t}^{2}=\epsilon_{0} \Delta \chi_{1} \omega_{\alpha} t s E_{s t}^{2} .
$$

We shall assume that the ions are dispersed homogeneously within the various DH's (the opposite case is treated in Sec. A 3). By solving the power balance equation that one writes for each DH $[42,43]$, one gets

$$
\delta T_{s t}=\tau_{\mathrm{DH}} \mathscr{P}_{s t} / c_{\mathrm{DH}} .
$$

Combining Eqs. (A1), (A3), and (A4), one can compare the heating produced by a static field and that produced by an ac field of equal amplitude

$$
\left(\frac{\delta T_{s t}}{\delta T_{a c}}\right)_{E_{a c}=E_{s t}}=2 \omega_{\alpha} \tau_{\mathrm{DH}} t s<5 t s \leqslant 5 \times 10^{-3},
$$

where the first inequality is obtained by replacing $\tau_{\mathrm{DH}}$ by its maximal value equals to $2.5 \tau_{\alpha}$ in glycerol [44]; and where the last inequality is obtained by using the maximal experimental values of the two small numbers $t$ and $s$ defined above. Even with these two successive overestimates, we find that $\delta T_{s t}$ is more than two orders of magnitude smaller than $\delta T_{a c}$.

\section{Comparison of the nonlinear polarizations produced either by $\boldsymbol{E}_{a c}$ or by $\boldsymbol{E}_{s t}$}

We now compare the nonlinear polarization $\delta P$ produced by the two kinds of heatings calculated in the previous subsection. For each DH one has a nonlinear polarization $\delta P_{\mathrm{DH}}$ given by [42]

$$
\delta P_{\mathrm{DH}}=\epsilon_{0} \frac{\partial \chi_{\mathrm{Lin}}}{\partial T} \delta T,
$$

where the heating $\delta T$ depends on the value of the relaxation time $\tau_{\mathrm{DH}}$ of each $\mathrm{DH}$, and is given either by Eq. (A1) or by Eq. (A4).

To obtain $\delta P$, one has to integrate Eq. (A6) over all the possible values of $\tau_{\mathrm{DH}}$ with a weight $\mathcal{G}\left(\tau_{\mathrm{DH}}\right) d \tau_{\mathrm{DH}}$. For the two reasons given above just after Eq. (A1), as soon as $\omega \geqslant 1.12 \omega_{\alpha}$, the dominant terms for the calculation of the nonlinear effects are given by the DH's such that $\omega \tau_{\mathrm{DH}} \geqslant 1$. We thus obtain

$$
\frac{\delta P_{s t}}{\delta P_{a c}} \simeq \frac{\int_{\tau \geqslant 1 / \omega} \frac{\partial \chi_{\mathrm{Lin}}}{\partial T} \delta T_{s t} \mathcal{G}\left(\tau_{\mathrm{DH}}\right) d \tau_{\mathrm{DH}}}{\int_{\tau \geqslant 1 / \omega} \frac{\partial \chi_{\mathrm{Lin}}}{\partial T} \delta T_{a c} \mathcal{G}\left(\tau_{\mathrm{DH}}\right) d \tau_{\mathrm{DH}}} .
$$

As the condition $\omega \tau_{\mathrm{DH}} \geqslant 1$ ensures that both the real and the imaginary parts of each integrand in Eq. (A7) do not change sign over the integration domain, we shall overestimate $\delta P_{s t} / \delta P_{a c}$ by using the maximal value of $\delta T_{s t}$ and the minimal value of $\delta T_{a c}$, i.e., $\delta T_{a c}\left(\omega \tau_{\mathrm{DH}}=1\right)$. We thus obtain by using Eqs. (A1) and (A5)

$$
\frac{\delta P_{s t}}{\delta P_{a c}}<10 t s<10^{-2}
$$

and therefore, taking into account the fact that the prefactors of the two terms of $\delta P(t)$ containing $\chi_{3}^{(1)}$ and $\chi_{2 ; 1}^{(1)}$ differ by a factor of 4 , we conclude that the box model predicts that, for $\omega \geqslant 1.12 \omega_{\alpha}$, the values of $\left|\chi_{2 ; 1}^{(1)}\right|$ are more than four hundred times smaller than those of $\left|\chi_{3}^{(1)}\right|$. This is contradicted by Fig. 5 of the main paper.

\section{Homogeneous heatings}

Contrary to the previous paragraphs where each $\mathrm{DH}$ has its own temperature, we shall consider here the supercooled liquid as being homogeneously heated by the dissipated electrical power. Here the term "homogeneous" refers to the fact that we assume that the value of dielectric susceptibility is the same in every point of the liquid, hence the volumic dissipated power is the same everywhere in the liquid. For a pure ac field, we have thoroughly studied this question of homogeneous heatings in Ref. [48]: the heat propagation equation was solved within the samples, assuming that the electrodes were thick enough to be ideal heat sinks (i.e., with a zero heating). It was found that below $204 \mathrm{~K}$, the nonlinear susceptibility due to homogeneous heatings were, at any frequency, more than 20 times smaller than the third harmonics cubic susceptibility $\chi_{3}^{(3)}$. In the range $204-225 \mathrm{~K}$, the heating contribution to $\chi_{3}^{(3)}$ increases but remains marginal as long as one does not consider angular frequencies much larger than $\omega_{\alpha}$, see Ref. [48].

For the reasons already explained just after Eq. (A2) above, only two terms matter for the dissipated power in a $\chi_{2 ; 1}^{(1)}$ experiment: the first term is $\mathscr{P}_{s t}=\sigma E_{s t}^{2}$ which is constant in time; the second term is $\mathscr{P}_{1 \omega}=2 \sigma E_{s t} E_{a c} \cos (\omega t)$ : it oscillates at $1 \omega$. As in the previous section, $\mathscr{P}_{s t}$ gives a first harmonics nonlinear polarization $\propto t s \ll 1$ much larger than the nonlinear polarization $\propto(t s)^{2}$ coming from $\mathscr{P}_{1 \omega}$. The fact that $\mathscr{P}_{1 \omega}$ is negligible is reinforced by the fact that it yields a homogeneous heating $\delta T_{1 \omega}^{\mathrm{hom}}$ which is confined to the glycerol samples due to the skin effect which prevents ac heatings from extending over long distances [48]. This contrasts with the homogeneous heating $\delta T_{s t}^{\text {hom }}$ coming from $\mathscr{P}_{s t}$ : as it is constant in time, it is not exponentially damped at long distances. Therefore, the corresponding dc heat flux crosses the-unavoidable-insulating piece of thickness $L_{\text {ins }}$ which ensures the absence of electrical contact between each electrode and the experimental cell. In our case, this insulating piece is a kind of polymeric resin which conducts heat with a thermal conductivity $\kappa_{\text {ins }}$, yielding

$$
\delta T_{s t}^{\mathrm{hom}} \simeq \sigma E_{s t}^{2} S e \frac{L_{\mathrm{ins}}}{\kappa_{\mathrm{ins}} S}=\epsilon_{0} \Delta \chi_{1} \omega_{\alpha} E_{s t}^{2} t s e L_{\mathrm{ins}} / \kappa_{\mathrm{ins}},
$$

where $e$ and $S$ are, respectively, the thickness and the surface of the sample (equal to that of the insulating piece).

We shall now contrast this expression of $\delta T_{s t}^{\text {hom }}$ with that of $\delta T_{a c}^{\text {hom }}$, where $\delta T_{a c}^{\text {hom }}$ is the homogeneous heating in an experiment with a pure ac field. Focusing, for simplicity to the 
case $\omega=\omega_{\alpha}$, we have [48], with $\chi^{\prime}\left(\omega_{\alpha}\right) \simeq 0.3 \Delta \chi_{1}$ :

$$
\delta T_{a c}^{\mathrm{hom}} \simeq 0.3 \epsilon_{0} \Delta \chi_{1} \omega_{\alpha} E_{a c}^{2} e^{2} / \kappa_{\mathrm{gly}},
$$

where $\kappa_{\mathrm{gly}}$ is the thermal conductivity of the glycerol. As in our case one has $\kappa_{\text {ins }} \approx \kappa_{\text {gly }}$ we obtain

$$
\frac{\delta T_{s t}^{\mathrm{hom}}}{\delta T_{a c}^{\mathrm{hom}}} \simeq \frac{t s L_{\mathrm{ins}}}{0.3 e} \leqslant 0.4,
$$

where the last inequality was obtained by putting the numbers corresponding to our experiment, e.g., $L_{\text {ins }}=3 \mathrm{~mm}$ and $e=$ $16 \mu \mathrm{m}$. We thus conclude that, despite its static character which enlarges its value, $\delta T_{s t}^{\text {hom }}$ is weaker than the homogeneous heating $\delta T_{a c}^{\text {hom }}$ obtained in a pure ac field experiment. Since, as long as $T \leqslant 225 \mathrm{~K}$, one finds [48] that $\delta T_{a c}^{\text {hom }}\left(\omega_{\alpha}\right)$ yields a nonlinear term more than four times smaller than $\left|\chi_{3}^{(3)}\left(\omega_{\alpha}\right)\right|$, and as $\left|\chi_{3}^{(3)}\left(\omega_{\alpha}\right)\right| \leqslant 0.1\left|\chi_{3}^{(1)}\left(\omega_{\alpha}\right)\right|$, we get with $\left|\chi_{3}^{(1)}\left(\omega_{\alpha}\right)\right| \approx$ $\left|\chi_{2 ; 1}^{(1)}\left(\omega_{\alpha}\right)\right|$ that $\delta T_{s t}^{\text {hom }}$ yields a first harmonics nonlinear term which is at least two orders of magnitude smaller than $\left|\chi_{2 ; 1}^{(1)}\left(\omega_{\alpha}\right)\right|$. Extending these arguments for all the frequencies and temperatures studied in the main paper, we find that $\delta T_{s t}$ yields a first harmonics nonlinear polarization smaller than the $\pm 4 \%$ accuracy corresponding to our experiments measuring $\chi_{2 ; 1}^{(1)}(\omega)$.

Note that it would be easy to design an experimental setup where $L_{\text {ins }}$ would be as small as $e$ : in that case one would have $\delta T_{s t}^{\text {hom }} \ll \delta T_{a c}^{\text {hom }}$. This shows that these $\chi_{2 ; 1}^{(1)}$ experiments could be done, in the stationary regime, up to very high temperatures without having any heating problem. This strikingly contrasts with the pure ac field nonlinear experiments, in the steady state regime, where heating becomes an issue as soon as $f_{\alpha}$ is above (typically) $10 \mathrm{kHz}$, see Ref. [48]. One could thus imagine first to "calibrate" the $\chi_{2 ; 1}^{(1)}$ temperature dependence over that of $\chi_{3}^{(1 \text { or } 3)}$ in the temperature range where heating effects are negligible, and then to push the $\chi_{2 ; 1}^{(1)}$ experiments to very high temperatures, taking advantage of the smallness of heatings with a static field. As stated in the main paper, this would allow to draw the temperature evolution of $N_{\text {corr }}$ in an unprecedentedly large temperature interval.

\section{APPENDIX B: DERIVATION OF THE TOY MODEL'S PREDICTION FOR $\chi_{2 ; 1}^{(1)}$}

In this section we shall study the "toy model" for the dielectric response close to the glass transition. This toy model was studied in Ref. [47] where its predictions for the cubic susceptibilities $\chi_{3}^{(1 \text { or } 3)}$ in a pure ac field experiment were computed in detail. This model expands on a previous work of Ref. [45] where each DH is represented as a point evolving in an asymmetric double well (ADW), independently of the state of all other DH's. In this model, the energy asymmetry $\Delta$ is the difference between the minimal energy in the two wells, the deeper well being labeled by the value of the angle $\theta_{1}$ between the net dipole moment $\mu$ of the considered $\mathrm{DH}$, and the direction of the applied external field. For simplicity, it is assumed that the angle between the dipole moment and the external applied field is $\theta_{2}=\theta_{1}+\pi$ in the well of high energy. The height of the barrier between the two wells, as well as $\Delta / k_{B} T$ with $k_{B}$ the Boltzmann's constant, sets the relaxation time $\tau \propto 1 / \cosh \left[\Delta /\left(2 k_{B} T\right)\right]$ of the corresponding $\mathrm{DH}$. The distribution of the $\tau^{\prime}$ s is assumed to be given by $\mathcal{G}(\tau)$ where $\mathcal{G}(\tau)$ is the suitable function allowing to recover the experimental frequency dependence of the macroscopic linear dielectric susceptibility. The decisive improvement made in Ref. [47] was to assume that the net dipole moment of any DH is given by $\mu=\mu_{\text {molec }} \sqrt{N_{\text {corr }}}$, with $\mu_{\text {molec }}$ the molecular dipole moment: this expresses the fact that no structural order exists in a given DH. This directly gives [47] that the macroscopic linear dielectric susceptibility $\chi_{\text {Lin }}$ is independent of $N_{\text {corr }}$, while the macroscopic cubic susceptibilities $\chi_{3}^{(1 \text { or } 3)}$ in a pure ac field experiment are directly proportional to $N_{\text {corr }}$.

We shall now compute the prediction of this toy model for $\chi_{2 ; 1}^{(1)}$. Let us first consider a set of $N$ identical ADW; i.e., a set of ADW's sharing the same values for all microscopic parameters of the model. When the formula for $\chi_{2 ; 1}^{(1)}$ in this subset of DH's will have been derived, we shall just have to integrate over the various values of $\theta_{1}$, and of $\tau$ with the function $\mathcal{G}(\tau)$.

We first remind the reader the basic equations for a pure ac field $E_{a c}$. Denoting $n_{1}\left(n_{2}\right)$ the number of ADW's in state $\theta_{1}\left(\theta_{2}=\theta_{1}+\pi\right)$, the polarization $P$ of the considered set of ADW's is, with $n=n_{1}-n_{2}$ :

$$
\begin{aligned}
P & =\frac{\left(n_{1}-n_{2}\right) \mu_{\text {molec }} \sqrt{N_{\text {corr }}} \cos \theta_{1}}{N N_{\text {corr }} a^{3}} \\
& =\mathcal{M} \frac{n}{N} \text { where } \mathcal{M}=\frac{\mu_{\text {molec }} \cos \theta_{1}}{\sqrt{N_{\text {corr }}} a^{3}} .
\end{aligned}
$$

Combining the two master equations for $n_{1}(t)$ and $n_{2}(t)$, with $n_{2}(t)=N-n_{1}(t)$, one gets the equation for the dynamics of $P$ :

$$
\tau \frac{d P}{d t}+P(\delta \sinh e+\cosh e)=\mathcal{M}(\delta \cosh e+\sinh e)
$$

where

$$
e(t) \equiv F \cos (\omega t), \quad F=\frac{\mu_{\mathrm{molec}} \sqrt{N_{\mathrm{corr}}} \cos \left(\theta_{1}\right)}{k_{B} T} E_{a c}
$$

and

$$
\mathcal{M}=\frac{\mu_{\text {molec }} \cos \theta_{1}}{\sqrt{N_{\text {corr }}} a^{3}}, \quad \delta=\tanh \left(\frac{\Delta}{2 k_{B} T}\right) .
$$

Adding a constant field $E_{s t}$ is equivalent to changing the asymmetry $\Delta$ by an amount $2 \mu_{\operatorname{molec}} \sqrt{N_{\operatorname{corr}}} E_{s t} \cos \theta_{1}$. This amounts to changing the relaxation time $\tau$ by an amount $\partial \tau$ and the reduced asymmetry $\delta$ by an amount $\partial \delta$, where the notation $\partial$ for differentiation was preferred to the usual $\delta$ to avoid the weird expression $\delta \delta$. By using the exponent "bare" to label (when necessary) the quantities when $E_{s t}=0$, we obtain

$$
\begin{aligned}
\partial \delta & =d_{1} G+d_{2} G^{2} \quad \text { with } \\
d_{1} & =\frac{1}{\cosh ^{2}\left(\frac{\Delta^{\text {bare }}}{2 k_{B} T}\right)} ; d_{2}=-d_{1} \delta \quad \text { and } \\
G & =\frac{\mu_{\text {molec }} \sqrt{N_{\text {corr }}} \cos \theta_{1}}{k_{B} T} E_{s t} \\
\partial \tau & =\tau^{\text {bare }}\left(\mathcal{T}_{1} G+\mathcal{T}_{2} G^{2}\right) \quad \text { with } \\
\mathcal{T}_{1} & =-\delta^{\text {bare }} \text { and } \\
\mathcal{T}_{2} & =-\frac{1}{2}\left[\frac{1}{\cosh ^{2}\left(\frac{\Delta^{\text {bare }}}{2 k_{B} T}\right)}-\left(\delta^{\text {bare }}\right)^{2}\right] .
\end{aligned}
$$


In Ref. [47] $P(t)$ was expanded in series of powers of the field $E_{a c}$ up to third order $P^{\text {bare }}(t)=P_{0}^{\text {bare }}+P_{1}^{\text {bare }}(t)+$ $P_{2}^{\text {bare }}(t)+P_{3}^{\text {bare }}(t)$ where $P_{q}^{\text {bare }} \propto\left(E_{a c}\right)^{q} \propto e^{q}$. This expansion was made iteratively taking advantage that in experiments $e \ll 1$, which allows to neglect all $P_{q^{\prime}>q}^{\text {bare }}$ terms when computing $P_{q}^{\text {bare }}$. As to compute $\chi_{2 ; 1}^{(1)}$ we are only interested in terms $\propto E_{a c} E_{s t}^{2}$, we have only to expand $P_{1}^{\text {bare }}(t)$ to second order in $E_{s t}$ to obtain all the terms contributing to $\chi_{2 ; 1}^{(1)}$. In Ref. [47] it was found that

$$
P_{1}^{\text {bare }}(t)=\frac{\mathcal{M}\left(1-\delta^{2}\right)}{\sqrt{1+(\omega \tau)^{2}}} F \cos [\omega t-\arctan (\omega \tau)],
$$

and when $E_{s t} \neq 0$ we thus have

$$
\begin{aligned}
P_{1}-P_{1}^{\text {bare }}= & \frac{\partial P_{1}^{\text {bare }}}{\partial \tau} \partial \tau+\frac{\partial P_{1}^{\text {bare }}}{\partial \delta} \partial \delta \\
& +\frac{1}{2} \frac{\partial^{2} P_{1}^{\text {bare }}}{\partial \tau^{2}} \partial \tau^{2}+\frac{1}{2} \frac{\partial^{2} P_{1}^{\text {bare }}}{\partial \delta^{2}} \partial \delta^{2} \\
& +\frac{\partial^{2} P_{1}^{\text {bare }}}{\partial \tau \partial \delta} \partial \partial \delta,
\end{aligned}
$$

which means that we have to compute the five terms of the right-hand side of Eq. (B5) to get all the terms contributing to $\chi_{2 ; 1}^{(1)}$. This can be done readily from Eqs. (B3) and (B4). Below we write only the relevant terms, i.e., we keep only the terms proportional to $G^{2} \propto E_{s t}^{2}$ in each of the five terms (the terms proportional to $G$ indeed vanish when averaging over $\theta_{1}$, see below). We obtain

$$
\begin{aligned}
\frac{\partial P_{1}^{\text {bare }}}{\partial \tau} \partial \tau & =\tau^{\text {bare }} \frac{\partial P_{1}^{\text {bare }}}{\partial \tau} \mathcal{T}_{2} G^{2}, \\
\frac{\partial P_{1}^{\text {bare }}}{\partial \delta} \partial \delta & =\frac{-2 \delta}{1-\delta^{2}} P_{1}^{\text {bare }} d_{2} G^{2}, \\
\frac{1}{2} \frac{\partial^{2} P_{1}^{\text {bare }}}{\partial \tau^{2}} \partial \tau^{2} & =\frac{1}{2} \frac{\partial^{2} P_{1}^{\text {bare }}}{\partial \tau^{2}}\left(\tau^{\text {bare }} \mathcal{T}_{1} G\right)^{2}, \\
\frac{1}{2} \frac{\partial^{2} P_{1}^{\text {bare }}}{\partial \delta^{2}} \partial \delta^{2} & =\frac{-1}{1-\delta^{2}} P_{1}^{\text {bare }} d_{1}^{2} G^{2}, \\
\frac{\partial^{2} P_{1}^{\text {bare }}}{\partial \tau \partial \delta} \partial \tau \partial \delta & =\frac{-2 \delta}{1-\delta^{2}} \tau^{\text {bare }} \frac{\partial P_{1}^{\text {bare }}}{\partial \tau} \mathcal{T}_{1} d_{1} G^{2} .
\end{aligned}
$$

As the previous calculations have been done for the DH's having the same relaxation time $\tau$, the expression of the complex linear dielectric susceptibility $\chi_{\mathrm{Lin}}^{\text {bare }}$ at $E_{s t}=0$ is drawn from Eq. (B4) by using $\chi_{\text {Lin }}^{\text {bare }}=P_{1}^{\text {bare }} /\left(\epsilon_{0} E_{a c}\right)$. We thus obtain a relation holding both for the real and the imaginary parts of $\chi_{2 ; 1}^{(1)}$ :

$$
\begin{aligned}
\chi_{2 ; 1}^{(1)}= & \frac{G^{2}}{3 E_{s t}^{2}}\left(\frac{1}{2} \frac{\partial^{2} \chi_{\text {Lin }}^{\text {bare }}}{\partial \tau^{2}}\left(\tau^{\text {bare }} \mathcal{T}_{1}\right)^{2}\right. \\
& \left.+\tau^{\text {bare }} \frac{\partial \chi_{\text {Lin }}^{\text {bare }}}{\partial \tau}\left(\frac{-2 \delta}{1-\delta^{2}} \mathcal{T}_{1} d_{1}+\mathcal{T}_{2}\right)+\chi_{\text {Lin }}^{\text {bare }} \frac{-2 \delta d_{2}-d_{1}^{2}}{1-\delta^{2}}\right) .
\end{aligned}
$$

As Eq. (B7) holds for a given value of $\tau$ and of $\theta_{1}$ [since $\theta_{1}$ is both in $G^{2}$ and in $\chi_{\mathrm{Lin}}^{\text {bare }}$ see Eqs. (B2)-(B4)], we have to average over these two quantities.
(1) Averaging over $\theta_{1}$ : As the wells are assumed to be infinitely deep (see Ref. [47]), we have to neglect the effect of $E_{s t}$ on the distribution of $\theta_{1}$ and to consider that $\theta_{1}$ is isotropically distributed whatever $E_{s t}$. Therefore the average over $\theta_{1}$ is straightforward, just as in Ref. [47]. By introducing $\mathcal{F}_{\text {Lin }}^{\text {bare }}=\chi_{\text {Lin }}^{\text {bare }} / \Delta \chi_{1}$ we obtain from Eq. (B7) and $\left\langle\cos ^{2} \theta_{1}\right\rangle_{\theta_{1}}=$ 1/3:

$$
\begin{aligned}
\left\langle\chi_{2 ; 1}^{(1)}\right\rangle_{\theta_{1}}= & \frac{\epsilon_{0}\left(\Delta \chi_{1}\right)^{2} a^{3}}{k_{B} T} \frac{N_{\text {corr }}}{3\left(1-\delta^{2}\right)}\left(\frac{1}{2} \frac{\partial^{2} \mathcal{F}_{\text {Lin }}^{\text {bare }}}{\partial \tau^{2}}\left(\tau^{\text {bare }} \mathcal{T}_{1}\right)^{2}\right. \\
& +\tau^{\text {bare }} \frac{\partial \mathcal{F}_{\text {Lin }}^{\text {bare }}}{\partial \tau}\left(\frac{-2 \delta}{1-\delta^{2}} \mathcal{T}_{1} d_{1}+\mathcal{T}_{2}\right) \\
& \left.+\mathcal{F}_{\text {Lin }}^{\text {bare }} \frac{-2 \delta d_{2}-d_{1}^{2}}{1-\delta^{2}}\right) .
\end{aligned}
$$

(2) Averaging over $\tau$ and $N_{\text {corr }}$ : Then, we have to average over the $\tau^{\prime}$ s with the weight $\mathcal{G}(\tau) d \tau$ already evoked above. Finally, we have to average over the various values of $N_{\text {corr }}$ which is likely to be distributed within the various DH's. We note $\left[N_{\text {corr }}\right]_{a v}$ the corresponding average value of $N_{\text {corr }}$ : as in Ref. [47] this yields the macroscopic $\chi_{2 ; 1}^{(1)}$ to be proportional to $\left[N_{\text {corr }}\right]_{a v}$. More precisely, moving to the dimensionless version $X_{2 ; 1}^{(1)}$ of $\chi_{2 ; 1}^{(1)}$ with the definition $X_{2 ; 1}^{(1)} \equiv \chi_{2 ; 1}^{(1)} k_{B} T /\left[\epsilon_{0}\left(\Delta \chi_{1}\right)^{2} a^{3}\right]$ we obtain

$$
X_{2 ; 1}^{(1)}=\frac{\left[N_{\text {corr }}\right]_{a v}}{3\left(1-\delta^{2}\right)} \mathcal{J}\left(\omega \tau_{\alpha}\right),
$$

where for brevity we have denoted $\mathcal{J}\left(\omega \tau_{\alpha}\right)$ the integral with weight $\mathcal{G}(\tau) d \tau$ of the expression written between large brackets in the right-hand side of Eq. (B8). Note that due to time temperature superposition (TTS), we expect that the function $\mathcal{J}\left(\omega \tau_{\alpha}\right)$ does not explicitly depends on the temperature. As in the toy model it is reasonable to choose that $\delta$ is a constant in temperature (see Ref. [47]), we conclude that this model predicts that the temperature evolution of $X_{2 ; 1}^{(1)}\left(f / f_{\alpha}\right)$ is directly that of $\left[N_{\text {corr }}(T)\right]_{a v}$. This is what is explained in the main paper, with the simplified notation $N_{\text {corr }}(T)$ instead of the more precise one $\left[N_{\text {corr }}(T)\right]_{a v}$.

Another important feature of Eq. (B7) is that the second term of the second factor of the right-hand side is proportional to $\partial \chi_{\text {Lin }}^{\text {bare }} / \partial T$. Indeed, we have

$$
\tau^{\text {bare }} \frac{\partial \chi_{\operatorname{Lin}}^{\text {bare }}}{\partial \tau}=\frac{\partial \chi_{\operatorname{Lin}}^{\text {bare }}}{\partial T} \frac{1}{\partial \ln \tau_{\alpha} / \partial T},
$$

where we have used the TTS property ensuring that all the various $\tau^{\prime}$ s have the same relative variation in temperature. Therefore, the fact that the toy model is able to fit our data of $\chi_{2 ; 1}^{(1)}$ is not so surprising since Eqs. (B7) and (B10) show that $\chi_{2 ; 1}^{(1)}$ is related to $\partial \chi_{\mathrm{Lin}}^{\text {bare }} / \partial T$. Of course these two quantities are proportional only when the first and the third terms of the second factor of the right-hand side of Eq. (B7) are negligible. It turns out that, numerically, these two terms play quite a small role. But their mere existence emphasizes the phenomenological nature of the toy model. As stated in the main paper, the ability of this model to fit our data does not change its phenomenological nature and that it cannot explain, from first principles, the existence of a deep relationship between $\chi_{2 ; 1}^{(1)}$ and $\partial \chi_{\text {Lin }}^{\text {bare }} / \partial T$. 
Two last points, mentioned in the main paper, are noteworthy.

(1) To take into account the finite lifetime $\tau_{\mathrm{ex}}$ of the DH's, we make a linear combination of the expression of $\chi_{2 ; 1}^{(1)}$ derived above and of $\chi_{2 ; 1 ; \text { triv }}^{(1)}$ corresponding to the "trivial" case of a ideal gas of dipoles. The expression of $\chi_{2,1 ; \text { triv }}^{(1)}$ is drawn from Ref. [29], and a global multiplicative factor close to 0.8 is allowed onto $\chi_{2 ; 1 ; \text { triv }}^{(1)}$, just as in Ref. [47]. For the same reasons as in Ref. [47], $\chi_{2 ; 1 ; \text { triv }}^{(1)}$ is weighted by the factor $1-\exp \left(-f_{\mathrm{ex}} / f\right)$ where $f_{\mathrm{ex}}$ is a phenomenological parameter of the order of $1 / \tau_{\mathrm{ex}}$. Of course, the expression drawn from Eq. (B7) is weighted by the complementary factor $\exp \left(-f_{\text {ex }} / f\right)$. As the best choice to fit our experiments turns out to be $f_{\text {ex }} \simeq 0.15 f_{\alpha}$ (just as in Ref. [47]), the influence of $\chi_{2 ; 1 ; \text { triv }}^{(1)}$ on the overall fit is really small as soon as $f \geqslant 0.5 f_{\alpha}$. Of course, $\chi_{2 ; 1 ; \text { triv }}^{(1)}$ plays a dominant role for $f \leqslant 0.1 f_{\alpha}$.

(2) One can average over $\delta$, which is likely to be distributed among the various DH's. As in Ref. [47], we have chosen either a single value of $\delta$, and the best value for fitting our data of $\chi_{2 ; 1}^{(1)}$ is $\delta=0.6$ just as in Ref. [47]; or we have taken a Gaussian expression with parameters $\delta_{1}$ and $\delta_{2}$. Note that, as $\delta$ is restricted over the $[0 ; 1]$ interval, $\delta_{1}$ and $\delta_{2}$ are in general neither the average of $\delta$ nor its standard deviation. Very surprisingly we found that the set of best values of $\delta_{1}$ and $\delta_{2}$ to fit our experiments are the same as those selected in Ref. [47] when fitting the data of $\chi_{3}^{(1 \text { or 3) }}$. The two continuous lines of Fig. 5 of the main letter correspond to the two kinds of fits $\left(\delta=0.6\right.$ single valued and $\left[N_{\text {corr }}\right]_{a v}=16$; or $\left[N_{\text {corr }}\right]_{a v}=10$ and $\delta$ distributed with the above-mentioned Gaussian distribution).
[1] Dynamical Heterogeneities in Glasses, Colloids and Granular Media, edited by L. Berthier, G. Biroli, J.-P. Bouchaud, L. Cipelletti, and W. van Saarloos (Oxford University Press, New York, 2010).

[2] A. Ikeda, L. Berthier, and P. Sollich, Soft Matter 9, 7669 (2013).

[3] M. D. Ediger, Annu. Rev. Phys. Chem. 51, 99 (2000).

[4] R. Richert, J. Phys.: Condens. Matter 14, R703 (2002).

[5] R. Candelier, O. Dauchot, and G. Biroli, Phys. Rev. Lett. 102, 088001 (2009).

[6] M. H. Cohen and D. Turnbull, J. Chem. Phys. 31, 1164 (1964).

[7] A. J. Liu and S. R. Nagel, Nature (London) 396, 21 (1998).

[8] M. Goldstein, J. Chem. Phys. 51, 3728 (1969).

[9] F. H. Stillinger, Science 267, 1935 (1995).

[10] P. G. Debenedetti and F. H. Stilinger, Nature (London) 410, 259 (2001).

[11] M. L. Ferrer, C. Lawrence, B. G. Demirjian, D. Kivelson, G. Tarjus, and C. Alba-Simionesco, J. Chem. Phys. 109, 8010 (1998).

[12] C. Alba-Simionesco, D. Kivelson, and G. Tarjus, J. Chem. Phys. 116, 5033 (2002).

[13] G. Tarjus, D. Kivelson, S. Mossa, and C. Alba-Simionesco, J. Chem. Phys. 120, 6135 (2004).

[14] C. M. Roland and R. Casalini, J. Chem. Phys. 121, 11503 (2004).

[15] G. Floudas, K. Mpoukouvalas, and P. Papadopoulos, J. Chem. Phys. 124, 074905 (2006).

[16] M. Tarzia, G. Biroli, J.-P. Bouchaud, and A. Lefèvre, J. Chem. Phys. 132, 054501 (2010).

[17] J.-P. Bouchaud and G. Biroli, Phys. Rev. B 72, 064204 (2005).

[18] L. Berthier, G. Biroli, J.-P. Bouchaud, L. Cipeletti, D. El Masri, D. L'Hôte, F. Ladieu, and M. Pierno, Science 310, 1797 (2005).

[19] C. Dalle-Ferrier, C. Thibierge, C. Alba-Simionesco, L. Berthier, G. Biroli, J.-P. Bouchaud, F. Ladieu, D. L'Hôte, and G. Tarjus, Phys. Rev. E 76, 041510 (2007).

[20] C. Thibierge, D. L'Hôte, F. Ladieu, and R. Tourbot, Rev. Scient. Instrum. 79, 103905 (2008).

[21] C. Crauste-Thibierge, C. Brun, F. Ladieu, D. L'Hôte, G. Biroli, and J.-P. Bouchaud, Phys. Rev. Lett. 104, 165703 (2010).
[22] C. Brun, F. Ladieu, D. L'Hôte, M. Tarzia, G. Biroli, and J.-P. Bouchaud, Phys. Rev. B 84, 104204 (2011).

[23] A. Drozd-Rzoska, S. J. Rzoska, M. Paluch, S. Pawlus, J. Ziolo, P. G. Santangelo, C. M. Roland, K. Czuprynski, and R. Dabrowski, Phys. Rev. E 71, 011508 (2005).

[24] A. Drozd-Rzoska, S. J. Rzoska, and J. Ziolo, Phys. Rev. E 77, 041501 (2008).

[25] For the first works about nonlinear dielectric effects, see J. Herweg, Z. Phys. 3, 36 (1920); A. Piekara and B. Piekara, C. R. Hebd. Seances Acad. Sci. 203, 852 (1936).

[26] R. Casalini, S. Capaccioli, S. Presto, M. Lucchesi, and P. A. Rolla, IEEE Trans. Diel. Elec. Ins. 8, 395 (2001).

[27] S. Hensel-Bielowka, S. Pawlus, C. M. Roland, J. Ziolo, and M. Paluch, Phys. Rev. E 69, 050501(R) (2004).

[28] A. Reiser, G. Kasper, and S. Hunklinger, Phys. Rev B 72, 094204 (2005).

[29] W. T. Coffey and B. V. Paranjape, Proc. Roy. Ir. Acad. 78A, 17 (1978); J. L. Déjardin, Yu. P. Kalmykov, Phys. Rev. E 61, 1211 (2000).

[30] As $N_{\text {corr }}$ increases when lowering $T$, we expect that the weight of the trivial effects in $\chi_{2 ; 1}^{(1)}$ gradually decreases, which could explain the possible slight increase of $\kappa$ and $\lambda_{\text {eff }}$ at low $T$-but this $T$ evolution of $\kappa, \lambda_{\text {eff }}$ is at the limit of our experimental uncertainty.

[31] P. M. Dejardin and F. Ladieu, J. Chem. Phys. 140, 034506 (2014).

[32] K. Schroter and E. Donth, J. Chem. Phys. 113, 9101 (2000).

[33] J. R. L. de Almeida and D. J. Thouless, J. Phys. A 11, 983 (1978).

[34] D. S. Fisher and D. A. Huse, Phys. Rev. B 38, 373 (1988); 38, 386 (1988).

[35] D. Petit, L. Fruchter, and I. A. Campbell, Phys. Rev. Lett. 83, 5130 (1999); 88, 207206 (2002).

[36] P. E. Jonsson, H. Takayama, H. A. Katori, and A. Ito, Phys. Rev. B 71, 180412(R) (2005).

[37] S. Miyashita, Proc. Jpn. Acad. Ser. B 86, 643 (2010).

[38] T. Bauer, P. Lunkenheimer, and A. Loidl, Phys. Rev. Lett. 111, 225702 (2013).

[39] G. Biroli and J.-P. Bouchaud, Physics 6, 128 (2013). 
[40] The closed symbols of the main part of Fig. 4 are obtained from the data on the inset after performing an average over $\left[2 f_{\alpha} ; 10 f_{\alpha}\right]$ to avoid "trivial contribution."

[41] The agreement is quantitative for $X_{3}^{(1)}$ —see Ref. [42]—and semiquantitative for $X_{3}^{(3)}$ - see Ref. [44].

[42] R. Richert and S. Weinstein, Phys. Rev. Lett. 97, 095703 (2006).

[43] W. Huang and R. Richert, Eur. Phys. J. B 66, 217 (2008).

[44] C. Brun, C. Crauste-Thibierge, F. Ladieu, and D. L'Hôte, J. Chem. Phys. 134, 194507 (2011).

[45] A. Wagner and H. Kliem, J. Chem. Phys. 111, 1043 (1999).
[46] R. Böhmer and G. Diezemann, in Broadband Dielectric Spectroscopy, edited by F. Kremer and A. Schönhals (Springer, New York, 2003), Chap. 14.

[47] F. Ladieu, C. Brun, and D. L'Hôte, Phys. Rev. B 85, 184207 (2012).

[48] C. Brun, C. Crauste-Thibierge, F. Ladieu, and D. L'Hôte, J. Chem. Phys. 133, 234901 (2010).

[49] Fields of $67 \mathrm{MV} / \mathrm{m}$ were used successfully in T. Bauer, P. Lunkenheimer, S. Kastner, and A. Loidl, Phys. Rev. Lett. 110, 107603 (2013). Note that this group now currently uses fields up to $80 \mathrm{MV} / \mathrm{m}$, still far from breakdown. 